Repository of the Max Delbrück Center for Molecular Medicine (MDC) Berlin (Germany)

\title{
Spontaneous self-assembly of pathogenic huntingtin exon 1 protein into amyloid structures.
}

Trepte, P., Strempel, N., Wanker, E.E. 


\section{Spontaneous self-assembly of pathogenic huntingtin exon 1 protein into amyloid structures}

Philipp Trepte, Nadine Strempel, Erich E. Wanker ${ }^{1}$

Neuroproteomics, Max Delbrueck Center for Molecular Medicine, Robert-Roessle-Str. 10,13125

Berlin, Germany

\section{Abstract:}

Polyglutamine (polyQ) diseases such as Huntington's (HD) or Spinocerebellar ataxia type 1 (SCA1) are neurodegenerative disorders caused by abnormally elongated polyQ tracts in human proteins. PolyQ expansions promote misfolding and aggregation of disease-causing proteins, leading to the appearance of nuclear and cytoplasmic inclusion bodies in patient neurons. Several lines of experimental evidence indicate that this process is critical for disease pathogenesis. However, the molecular mechanisms underlying spontaneous polyQ-containing aggregate formation and the perturbation of neuronal processes are still largely unclear.

This chapter reviews the current literature regarding misfolding and aggregation of polyQ-containing disease proteins. We specifically focus on studies that have investigated the amyloidogenesis of polyQ-containing huntingtin exon 1 (HTTex1) fragments. These protein fragments are disease-relevant and play a critical role in HD pathogenesis. We will outline potential mechanisms behind mutant HTTex1 aggregation and toxicity, as well as proteins and small molecules that can modify HTTex1 amyloidogenesis in vitro and in vivo. The potential implications of such studies for the development of novel therapeutic strategies are discussed.

\footnotetext{
${ }^{1}$ To whom correspondence should be addressed (email: ewanker@mdc-berlin.de)
} 


\section{Introduction:}

Polyglutamine (polyQ) tracts with lengths between 5 and 30 glutamines are conserved and found in $0.34 \%$ of all human proteins, indicating that they play an important functional role [1]. This view is supported by systematic computational and experimental investigations of polyQ-containing proteins, which suggest that polyQ domains mediate protein-protein interactions [1]. For example, polyQ sequences in transcription factors can influence gene expression in yeast and mammalian cells by promoting the formation of regulatory protein complexes that facilitate transcriptional activation [2-4]. Thus, polyQ sequences form functionally relevant protein domains that play a critical role in the assembly and disassembly of protein complexes.

However, substantial experimental evidence demonstrates that abnormally expanded polyQ tracts in proteins are toxic for cells and can cause severe inherited human diseases [4]. Such polyQ expansions are the result of rare genetic mutations, which were identified in patient families through positional cloning approaches [5]. The neurodegenerative disease Huntington's chorea is caused by a CAG trinucleotide expansion in the HTT gene, which leads to the synthesis of an elongated polyQ tract within the corresponding huntingtin (HTT) protein [6]. Wildtype, non-pathogenic HTT contains 6-35 glutamines, while the mutant, pathogenic protein in patients harbors more than 40 glutamines [6]. The polyQ tract in HTT is located at the $\mathrm{N}$-terminus, followed by a proline-rich region and three conserved HEAT repeats, which have a typical $\alpha$-helical solenoid structure [46]. Both the proline-rich and the HEAT repeat regions in HTT are critical for the assembly of protein complexes in neuronal cells [46, 47]. HTT is a large protein with a predicted molecular mass of about $350 \mathrm{kDa}$. Currently, its normal function in cells is not fully understood. However, studies in cell models and transgenic animals indicate that wild-type HTT plays a functional role in vesicle transport processes, cell signaling and transcriptional gene regulation, suggesting that it is a multifunctional scaffold protein that influences various cellular processes [48].

Huntington's disease (HD) is the most frequent form of the hereditary choreas. It has a multifaceted phenotype, including cognitive, psychiatric and motor impairments [6]. Symptoms of HD commonly become noticeable between the ages of 35-50 years; however, they can begin at any age from childhood to old age. HD affects the whole brain but certain areas such as the caudate nucleus and the putamen are most vulnerable. In these areas predominantly striatal medium spiny 
neurons are degraded, which play a key role in the control of movement and behavior [8].

To this day, 10 inherited polyQ expansion diseases have been reported, as summarized in Table 1. Although cellular dysfunction and toxicity are predominantly observed in neurons of the central nervous system (Table 1), the disease-causing proteins are ubiquitously expressed in all human tissues. Differentiated postmitotic neurons seem significantly more vulnerable to proteins with pathogenic polyQ tracts than fast-dividing mitotic cells. The molecular basis for the selective degeneration of neuronal cells in polyQ diseases, however, is still unclear [7].

Neurodegenerative polyQ expansion diseases are characterized by the accumulation of insoluble protein aggregates in neuronal cells (Table 1). These aggregates, which are often concentrated in large inclusion bodies, are observed in brain regions that display massive neurodegeneration, suggesting that the process of polyQ-mediated protein misfolding and aggregation drives pathogenesis [8]. This hypothesis is supported by investigations in transgenic mouse, fly and worm models, which indicate that toxicity in neuronal cells correlates with the formation of polyQcontaining protein aggregates [9]. Moreover, in vitro studies with polyQ disease proteins have demonstrated that both spontaneous protein aggregation and toxicity are dependent on polyQ length. Proteins with short, non-pathogenic polyQ tracts remain soluble, while proteins with long, pathogenic polyQ tracts self-assemble into insoluble, fibrillar protein aggregates or amyloids [10;45]. Hence, the formation of amyloidogenic protein aggregates is highly likely to be an important pathobiological process.

In this chapter, we will mainly review protein aggregation studies that focus on polyQ-containing disease-relevant N-terminal HTT exon 1 (HTTex1) fragments. Such protein fragments are generated in HD patients through the aberrant splicing of HTT mRNA [11]. HTTex1 rapidly self-assembles into protein aggregates in cell-free as well as cell-based assays and induces toxicity in various in vivo disease model systems [9;10]. A better understanding of the mechanism of HTTex1 aggregation and its impact on biological systems is critical for a better understanding of pathogenesis in $\mathrm{HD}$ and other polyQ disorders. 


\section{Pathogenic polyQ-containing protein aggregates in patients and disease models}

The deposition of $\mathrm{N}$-terminal HTT protein fragments with expanded polyQ sequences in neuronal inclusion bodies (IBs) is one pathological characteristic of HD brains $[8,9]$. IBs, which generally have a diameter of 1-5 $\mu \mathrm{m}$, are predominantly detected in cortical and striatal neurons [12;45]. Immunohistological studies revealed that IBs are exclusively detected with anti-HTT antibodies raised against $\mathrm{N}$-terminal HTT regions [13], suggesting that the truncated fragments rather than the full-length protein form insoluble disease-relevant protein aggregates in patient brains. Strikingly, such IBs are predominantly detected in the nuclei of neuronal cells, implying that the nuclear environment promotes the aggregation of the pathogenic protein (Fig. 1A) [8]. It is currently assumed that the misfolding and aggregation of mutant HTT recruits cellular proteins such as ubiquitin, molecular chaperones or components of the ubiquitin-proteasome system into IBs and thereby causes a redistribution of important functional proteins [12]. This might lead to the loss of function of multiple cellular pathways that depend on low-abundance proteins, such as transcription factors or molecular chaperones. Accordingly, neuronal IBs consisting of truncated, aggregated HTT fragments and multiple other cellular proteins are distinct subcellular structures that are exclusively detectable in the brains of patients and HD models with disease phenotypes.

Aggregation-prone HTT fragments also form soluble protein aggregates in neuronal cells [14]. These fibrillar oligomers or protofibrils, are diffusible structures (Fig. 1B) that can cause abnormal protein-protein interactions with other cellular proteins or lipids $[15 ; 16]$. This may result in the co-precipitation of metastable, natively unfolded proteins and perturb the membrane integrity of cells or transport vesicles [15;17].

In vitro and cell-based studies with polyQ-containing N-terminal HTT fragments showed that HTTex1 fragments with pathogenic polyQ tracts spontaneously self-assemble into insoluble protein aggregates with a $\beta$-sheet-rich, fibrillar morphology (Fig. 1B and C) [10;45]. Therefore, HTT aggregation is reminiscent of the amyloid- $\beta$ and $\alpha$-synuclein fibrillogenesis observed in the brains of patients with Alzheimer's and Parkinson's disease, respectively [18]. Strikingly, 
detailed investigations of HTTex1 fragments with different polyQ tracts have revealed that spontaneous HTTex1 fibrillogenesis is a polyQ-length dependent process [10]. HTTex1 fragments with non-pathogenic polyQ tracts are soluble, but large aggregate structures are detected in association with pathogenic HTTex1 fragments (Fig. 2A). Thus the polyQ-length-dependent aggregation of HTTex1 in vitro mirrors observations in patients and in transgenic model systems [8,9].

\section{Mechanism of polyQ-mediated HTT exon1 protein aggregation}

Studies with amyloidogenic polypeptides and proteins such as IAPP, A $\beta, \alpha-$ synuclein or PrP have demonstrated that the process of fibril self-assembly is generic and can be divided in two phases: (1) a lag phase where few or no fibrils form and (2) a fibril growth phase where a dramatic exponential increase in fibril mass is observed [19]. Usually, such spontaneous amyloid polymerization reactions have been explained with nucleation-dependent polymerization models [10;19;45]. These theoretical models assume that the rate-limiting step in the amyloidogenic pathway is the formation of a "nucleus", which develops slowly during the lag phase of the fibril assembly cascade. The "nucleus" is an oligomeric aggregate species of low abundance that is kinetically unstable and assembles with low propensity. Once formed, however, it rapidly grows into larger amyloid fibrils through an addition of monomers [10;19]. Thus the characteristic sigmoidal fibrillar growth profile observed for many amyloidogenic polypeptides reflects the greater ease by which monomers are added onto existing aggregates compared to the de novo formation of amyloidogenic oligomers (nuclei) from monomers through primary homogenous nucleation [19].

Spontaneous HTTex1 fibrillogenesis in vitro critically depends on the length of the polyQ tract (Fig. 2A) as well as on protein concentration and time (Fig. 2B). Moreover, aggregation can be stimulated by preformed fibrils, indicating that HTTex1 fibrillogenesis is dominated by a nucleation-dependent mechanism similar to that of other amyloidogenic polypeptides [10;45]. However, the details of the molecular mechanisms by which disease-relevant polyQ-containing HTTex1 fragments selfassemble into amyloid structures are currently not very well understood. Biochemical and biophysical studies hint that HTTex1 fragments with pathological polyQ tracts are converted into mature amyloid fibrils via spherical oligomers and/or protofibrils 
[14;20]. These structures were indeed detected in cell-free aggregation reactions by atomic force microscopy (AFM) [20]. However, these studies also provided experimental evidence that the vast majority of spontaneously forming HTTex1 fibrils are obtained through addition of monomers rather than through precursor oligomers [20]. Further studies are necessary to elucidate the critical steps in the HTTex1 polymerization cascade.

\section{Proteotoxicity of polyQ-containing protein aggregates}

Neurodegenerative disorders caused by CAG repeat expansions share two main characteristics: the deposition of protein aggregates and neuronal cell death. However, the identification of a defined neurotoxic aggregate species and the mechanism by which it ultimately causes toxicity is challenging and remains elusive.

Different types of mutant HTT (mHTT) aggregates such as oligomers, fibrils and IBs have been identified in neuronal cells [10;14;20;23]. IBs composed of mHTT fragments trap multiple proteins such as ubiquitin and chaperones, thereby exerting constant stress on the cellular environment [12;17]. Nevertheless, a number of studies indicate that IBs are less toxic for mammalian cells than small, soluble HTT protein aggregates. According to the evidence provided, $\mathrm{MHTT}$ fragments are transported into distinct, IB-like quality control compartments that accumulate the misfolded polyQ protein. Such compartments include structures such as the aggresome, the IPOD (insoluble protein deposit), the JUNQ (juxta- nuclear quality control) or the StiF (Sti1-inducible foci) [12;21;22]. Interestingly, the maturation of these IB-like compartments is regulated by cytosolic chaperones and their cochaperones, suggesting that chaperone pathways control the toxic effects of misfolded proteins in mammalian cells. Therefore, improving cells' capacity to deal with misfolded proteins by increasing the levels of molecular chaperones should reduce $\mathrm{mHTT}$-induced toxicity. Indeed, observations in mammalian and yeast cells have been made that the molecular chaperones $\mathrm{Hsc70}$ and Sti1p promote the accumulation of $\mathrm{mHTT}$ fragments into IB-like compartments [22;23]. This ultimately leads to reduced toxicity, suggesting that the process of the chaperone-induced formation of IBs is a strategy to protect cells against misfolded, proteotoxic polyQ proteins. Together, these studies imply that IBs, at least in rapidly dividing cells, have 
a protective role by serving as compartments for misfolded, aggregation-prone proteins. Even though IBs consisting of insoluble HTT aggregates are considered less harmful by some investigators than soluble aggregate species, they still exert a constant stress on protein homeostasis in mammalian cells. Recently, an artificial $\beta$ sheet-rich polypeptide, which forms typical amyloid fibrils in in vitro aggregation assays, was shown to be highly toxic for mammalian cells [23]. This polypeptide is thought to cause cellular toxicity through the formation of stable $\beta$-sheet-rich protein aggregates that can sequester several important, metastable proteins. PolyQ aggregates in cells potentially cause dysfunction and toxicity by binding essential cellular proteins, thereby preventing their ability to perform their normal cellular tasks [23]. However, the relevance of different aggregate species to pathogenesis needs further elucidation.

Mitotic cells have the ability to asymmetrically distribute inclusion bodies to only one of the daughter cells, allowing the other to develop free of aggregates. Cells left with IBs show a reduced capacity for reproduction, suggesting that they experience increased cellular stress [7]. As neurons are post-mitotic cells, they are constantly exposed to IBs and thus to proteotoxic stress. This is likely to lead to the dysfunction of key cellular pathways and the accumulation of additional misfolded proteins in a process that may last for decades. Numerous studies have analyzed the effects of polyQ-containing HTT fragments on a range of distinct cellular pathways (review in [4]). For example, mHTT fragments disrupt autophagy and the ubiquitin proteasome system (UPS) in cells, indicating that components of the cells' protein degradation-machinery are specifically vulnerable to polyQ aggregates [12;24;25]. In human HD brains, neuronal autophagosomes are enlarged and accumulate, supporting the view that $\mathrm{mHTT}$ aggregates perturb protein degradation pathways. In addition, reduced cytosolic turnover of $\mathrm{mHTT}$ aggregates has been observed in cell models. This phenomenon is thought to be the result of the defective recognition of HTT aggregates rather than of impaired autophagosome-lysosome fusion [24]. Interestingly, mHTT fragments are also present in synaptic terminals in neurons, where they form aggregates and cause an impairment of the local ubiquitinproteasome system (UPS) [25]. As the UPS is a critical modulator of synaptic plasticity and function, this highlights the importance of synaptic pathology in HD. Additionally, pathogenic HTT fragments disrupt axonal transport in neuronal cells. Under pathological conditions, the transport protein kinesin binds to microtubules 
with reduced affinity [26]. This is due to increased phosphorylation by the axonal cJun N-terminal kinase JNK3, which is hyperactivated by $\mathrm{mHTT}$ in neuronal cells. This disruption of the axonal transport machinery ultimately leads to an undersupply of synapses with essential membrane-bound cargos and thus to an impairment of synaptic function [26].

Previous studies have also suggested that $\mathrm{mHTT}$ aggregates are toxic for cells because they directly produce reactive oxygen species [27]. Similar results have also been reported for amyloid- $\beta$ aggregation reactions, supporting the observations with aggregation-prone HTTex1 fragments [28]. Intriguingly, experimental evidence suggests that aggregates formed of simple polyQ peptides are cytotoxic for mammalian cells when transported into the nucleus [29]. This suggests that the nuclear environment is especially vulnerable to polyQ protein aggregates. This could be due to the fact that polyQ aggregates recruit other polyQ or $Q$-rich proteins such as the TATA-binding protein (TBP), which subsequently lose their normal cellular functions [30]. Finally, evidence demonstrates that in vitro-produced fibrillar HTTex1 aggregates, rather than oligomers or monomers, are toxic for mammalian cells [15]. One potential mechanism for this behavior is that amyloid-like polyQ-containing protein aggregates can disrupt cellular membranes, potentially resulting in an uncontrolled breakage of cells or subcellular compartments [15]. These findings suggest that both small, diffusible $\mathrm{mHTT}$ aggregates and large IBs containing insoluble protein aggregates are harmful for cells. However, additional studies with disease-relevant model systems such as induced pluripotent stem (iPS) cell-derived neurons from HD patients need to be performed in order to elucidate the details of the molecular mechanisms by which polyQ protein aggregates perturb cellular systems.

\section{Modulation of polyQ-mediated protein aggregation by distinct cellular pathways and proteins}

Numerous proteins that modulate $\mathrm{mHTT}$ aggregation and toxicity in cell-free or cell-based assays have been identified [31;32]. Molecular chaperones and chaperone-associated proteins can directly bind to polyQ proteins. They can either stabilize the conformation and prevent the conversion of poly $Q$ sequences from a 
random coil into an aggregation-prone $\beta$-sheet-rich structure [31], or induce the degradation of the polyQ protein [32]. The chaperone CHIP, which possesses E3 ubiquitin ligase activity, binds to proteins with expanded polyQ tracts including $\mathrm{mHTT}$, which leads to an increased ubiquitination and degradation of the targeted protein [32]. Protein aggregates can also be cleared from cells via macro- or chaperonemediated autophagy (CMA). Selective catabolism in CMA is conferred by the presence of a KFERQ-like targeting motif in polyQ proteins, by which molecular chaperones recognize the hydrophobic surfaces of the misfolded substrates and transfer them to the lysosomal membrane protein LAMP-2A. They are then taken up into lysosomes, where they are degraded by lysosomal enzymes [33]. A synthetic fusion-peptide consisting of an Hsc70-binding motif and two polyQ-binding sequences can promote the degradation of mHTT through CMA [33]. The peptide activates CMA by stimulating the association of mHTT with the chaperone machinery. Enhanced clearance of mHTT fragments through treatment with this construct was observed in cell models as well as transgenic mice [33]. A similar strategy was applied to promote the degradation of polyQ aggregates through macroautophagy. In this case, a synthetic peptide derived from the key autophagy protein beclin1 was used to induce protein degradation [34]. Thus the development of autophagy-stimulating peptides is a powerful strategy to promote the clearance of polyQ-containing protein aggregates from mammalian cells.

For sake of completeness, it should be stated that posttranslational modifications such as phosphorylation, acetylation or SUMOylation influence the aggregation propensity of polyQ-containing HTT fragments in various model systems. Acetylation at lysine 444 (K444) increases the clearance of mHTT through macroautophagy. Expression of an acetylation-resistant mHTT fragment led to a dramatic increase in aggregation and neurodegeneration in primary cortical neurons and mouse brain [35]. Taken together, autophagy, chaperones and posttranslational modifications dramatically influence the biological activity of $\mathrm{mHTT}$ and its aggregation propensity in cells. 


\section{Identification of small molecules that influence polyQ-mediated protein aggregation}

Cellular processes that maintain proteostasis are of critical importance with regard to aggregation of polyQ-containing disease proteins. Proteostasis is maintained and regulated by molecular chaperones, autophagy, the ubiquitin proteasome system, and stress signaling pathways that sense the accumulation of abnormally folded proteins in cells [4;31-34]. Modulation of proteostasis is believed to have a powerful influence on misfolding and proteotoxicity of aggregation-prone disease proteins. Studies with the small molecule geldanamycin (GA), a potent inhibitor of Hsp90 ATPase activity, have revealed that increases in the levels of molecular chaperones such as Hsp70 or $\mathrm{Hsp} 40$ are associated with reduced aggregation and toxicity of mutant HTTex1 fragments in cells and flies [36]. The importance of chaperone networks in managing misfolded proteins in cells is also supported by the effect of the compound YM-1, which increases the binding affinity of Hsp70 to polyQ disease proteins, leading to an increased efficiency of their ubiquitination and degradation [37]. High-throughput screenings have led to the identification of numerous novel small molecules that influence the expression of molecular chaperones in mammalian cells [38]. The mechanism of action of these compounds needs to be further investigated, however, to develop new therapeutic strategies.

Small molecules that induce autophagy might also be of therapeutic value in the treatment of polyQ diseases. The well-known inducer rapamycin, an inhibitor of the mTOR pathway, potently decreases the abundance of insoluble polyQ HTT aggregates in cell models of HD and transgenic flies [39]. Rapamycin has only a mild effect in neurons, while the compound 10-NCP, an Akt inhibitor, was found to induce autophagy in neuronal cells more potently, suggesting that different compound classes may be required to promote the degradation of poly $Q$ aggregates in different cell types [40]. Besides small molecule promoters of functional proteostasis, compounds that directly target the aggregation process have been identified. They include substances such as Congo red, Thioflavine S, PGL-135 or EGCG ((-)epigallocatechin-3-gallate), which reduce the formation of polyQ-containing HTTex1 aggregates [41-43]. To date, the mode of action of EGCG has been studied most extensively (Fig. 3A). It directly binds to soluble HTTex1 fragments, reducing their 
propensity to spontaneously convert into $\beta$-sheet-rich fibrillar protein aggregates. Interestingly, EGCG does not block HTTex1 aggregation in cell-free assays. Rather, it promotes the formation of amorphous HTTex1 protein aggregates which are not observed in the absence of the substance [43]. This indicates that EGCG redirects the polyQ-mediated HTTex1 aggregation pathway, leading to the formation of a new type of aggregate structure. Studies in cells have revealed that this new type of HTTex 1 protein aggregate can be degraded more efficiently than $\beta$-sheet-rich fibrillar aggregates, resulting in decreased toxicity for mammalian cells.

Several studies indicate that critical features of neurodegenerative diseases such as protein misfolding, aggregation and neurotoxicty can be reproduced in transgenic fly models [49]. These investigations motivated us to assess the effect of EGCG on photoreceptor neurodegeneration in HD transgenic flies, overproducing an aggregation-prone HTTex1 protein with a pathogenic polyQ tract of 93 glutamines (HTTex1Q93). In this model the expression of HTTex1Q93 causes the progressive disruption of the regular trapezoidal arrangement of seven visible photoreceptor neurons (rhabdomers) that can be monitored by light microscopy. We found that in the absence of EGCG, HTTex1Q93-expressing neurons deteriorate until on average $\sim 3.5$ photoreceptors per ommatidium remain after 7 days (Fig. 3B). In EGCG treated flies, however, neurodegeneraton was significantly diminished, indicating that the compound has a protective effect on neurotoxicity in transgenic flies [43]. Intriguingly, EGCG is also a potent inhibitor of $\alpha$-synuclein and amyloid- $\beta$ fibrillogenesis, suggesting that it is a generic modulator of protein misfolding and aggregation [44]. We propose that EGCG functions as a chemical chaperone that can target multiple aggregation-prone proteins in a way similar to that of protein chaperones. In summary, targeting aggregation-prone proteins directly with small molecules might be an avenue for therapeutic interventions in polyQ and, more broadly, in protein misfolding diseases.

\section{Conclusions}

Polyglutamine diseases are caused by a CAG-expansion mutation in a disease gene, which leads to an elongated polyQ sequence in the protein product. HTTex1 containing a pathogenic polyQ stretch self-assembles and forms aggregates of 
different morphologies in vitro and in vivo. Similar to other amyloidogenic proteins, HTTex1 fibrillogenesis follows a polyQ-length-dependent nucleation and polymerization pathway. Controlled by the proteostasis network, cells have developed defense mechanisms, which inhibit misfolding and facilitate aggregate clearance. A better understanding of the basic principles of protein misfolding and aggregation will be the basis for the identification of small molecules which may be applicable as therapeutic agents.

\section{Summary}

- PolyQ-diseases are neurological disorders that are caused by a polyQ expansion mutation

- The expansion of the polyQ tract in the disease protein leads to misfolding and intracellular aggregate formation associated with cell-type-specific neurotoxicity and brain-region-specific atrophy

- HTTex1 spontaneously self-assembles into amyloid structures

- The aggregation reaction follows a nucleation-dependent mechanism

- PolyQ aggregates are $\beta$-sheet rich structures

- Intracellular inclusion bodies sequester a wide variety of cellular proteins leading to a disturbance of proteostasis

- Small diffusible mHTT aggregates as well as large inclusion bodies are harmful for cells

- There is no effective treatment for any of the polyQ diseases

\section{Key words}

- polyQ diseases

- Huntington's disease

- Neurodegenerative diseases

- Protein aggregation

- Inclusion bodies

- Nucleation-dependent polymerization

- $\beta$-sheet

- Chaperones

- Small molecules 


\section{Biographical Note}

- Erich Wanker is Chair of Molecular Medicine at Charité University Medicine Berlin and heads the Neuroproteomics research group at the Max Delbrück Center for Molecular Medicine Berlin-Buch. He graduated in Chemical Engineering and Biochemistry from the University of Technology Graz, Austria, where he also completed his $\mathrm{PhD}$ in 1992. After a postdoctoral fellowship at the University of California, Los Angeles he became a group leader at the MaxPlanck-Institute for Molecular Genetics Berlin and was appointed to his present positions in 2001. His research interests are in protein misfolding and neurodegeneration, molecular mechanisms of protein-protein and protein-drug interactions and on high-throughput network biology.

- Philipp Trepte is currently doing his PhD in the lab of Erich Wanker, working on the systematic generation and characterization of a protein interaction map of synaptic proteins. During his master' degree in Cell Biology at the University of Osnabrück, Germany, he visited the lab of Douglas Cyr at the UNC School of Medicine, USA, before he joined the lab of Philipp Khaitovich at the PICB, Shanghai, China for his master's thesis. His research interests focus on proteinprotein interactions, neurodegenerative diseases and synapse biology.

- Nadine U. Strempel is also working towards her PhD in the lab of Erich Wanker with a focus on the molecular mechanisms of huntingtin aggregation and its modulation by small molecules. Before coming to the Max Delbrück Center, she completed a diploma degree with a thesis in molecular biology and virology at the Department of Applied Tumor Virology of the German Cancer Research Center in Heidelberg. To deepen her knowledge in immunology and biochemistry, she studied one semester abroad at the University of Copenhagen, Denmark. 


\section{References}

[1] Schaefer et al. Evolution and function of CAG/polyglutamine repeats in protein-protein interaction networks. Nucleic Acids Research (2012) vol. 40 (10) pp. 4273-87

[2] Atanesyan et al. Polyglutamine tracts as modulators of transcriptional activation from yeast to mammals. Biological Chemistry (2012) vol. 393 (1-2) pp. 63-70

[3] Kim et al. Functional conservation of the glutamine-rich domains of yeast Gal11 and human SRC-1 in the transactivation of glucocorticoid receptor Tau 1 in Saccharomyces cerevisiae. Mol Cell Biol (2008) vol. 28 (3) pp. 913-25

[4] Hands et al. Polyglutamine gene function and dysfunction in the ageing brain. Biochim Biophys Acta (2008) vol. 1779 (8) pp. 507-21

[5] Gusella. Location cloning strategy for characterizing genetic defects in Huntington's disease and Alzheimer's disease. FASEB J (1989) vol. 3 (9) pp. 2036-41

[6] The Huntington's Disease Collaborative Research Group. A novel gene containing a trinucleotide repeat that is expanded and unstable on Huntington's disease chromosomes. Cell (1993) vol. 72 (6) pp. 971-83

[7] Lindner et al. Asymmetric segregation of protein aggregates is associated with cellular aging and rejuvenation. Proc Natl Acad Sci USA (2008) vol. 105 (8) pp. 3076-81

[8] Difiglia et al. Aggregation of huntingtin in neuronal intranuclear inclusions and dystrophic neurites in brain. Science (1997) vol. 277 (5334) pp. 1990-3

[9] Davies et al. Formation of neuronal intranuclear inclusions underlies the neurological dysfunction in mice transgenic for the HD mutation. Cell (1997) vol. 90 (3) pp. 537-48

[10]Scherzinger et al. Self-assembly of polyglutamine-containing huntingtin fragments into amyloid-like fibrils: implications for Huntington's disease pathology. Proc Natl Acad Sci USA (1999) vol. 96 (8) pp. 4604-9

[11]Sathasivam et al. Aberrant splicing of HTT generates the pathogenic exon 1 protein in Huntington disease. Proc Natl Acad Sci USA (2013) vol. 110 (6) pp. 2366-70

[12]Waelter et al. Accumulation of mutant huntingtin fragments in aggresome-like inclusion bodies as a result of insufficient protein degradation. Mol Biol Cell (2001) vol. 12 (5) pp. 1393-407

[13]Sieradzan et al. Huntington's disease intranuclear inclusions contain truncated, ubiquitinated huntingtin protein. Exp Neurol (1999) vol. 156 (1) pp. 92-9

[14]Sahl et al. Cellular inclusion bodies of mutant huntingtin exon 1 obscure small fibrillar aggregate species. Sci Rep (2012) vol. 2 pp. 895

[15]Pieri et al. Fibrillar $\alpha$-synuclein and huntingtin exon 1 assemblies are toxic to the cells. Biophys J (2012) vol. 102 (12) pp. 2894-905

[16]Harjes und Wanker. The hunt for huntingtin function: interaction partners tell many different stories. Trends Biochem Sci (2003) vol. 28 (8) pp. 425-33

[17] Olzscha et al. Amyloid-like aggregates sequester numerous metastable proteins with essential cellular functions. Cell (2011) vol. 144 (1) pp. 67-78

[18]Ross und Poirier. Protein aggregation and neurodegenerative disease. Nat Med (2004) vol. 10 Suppl pp. S10-7

[19]Jarrett und Lansbury. Seeding "one-dimensional crystallization" of amyloid: a pathogenic mechanism in Alzheimer's disease and scrapie?. Cell (1993) vol. 73 (6) pp. 1055-8

[20]Wacker et al. Hsp70 and Hsp40 attenuate formation of spherical and annular polyglutamine oligomers by partitioning monomer. Nature Structural \& Molecular Biology (2004) vol. 11 (12) pp. 1215-22 
[21] Kaganovich et al. Misfolded proteins partition between two distinct quality control compartments. Nature (2008) vol. 454 (7208) pp. 1088-1095

[22] Wolfe et al. The Hsp70/90 cochaperone, Sti1, suppresses proteotoxicity by regulating spatial quality control of amyloid-like proteins. Mol Biol Cell (2013) pp.

[23] Olshina et al. Tracking mutant huntingtin aggregation kinetics in cells reveals three major populations that include an invariant oligomer pool. J Biol Chem (2010) vol. 285 (28) pp. 21807-16

[24]Martinez-Vicente et al. Cargo recognition failure is responsible for inefficient autophagy in Huntington's disease. Nat Neurosci (2010) vol. 13 (5) pp. 567-76

[25]Wang et al. Impaired ubiquitin-proteasome system activity in the synapses of Huntington's disease mice. The Journal of Cell Biology (2008) vol. 180 (6) pp. 1177-89

[26]Morfini et al. Pathogenic huntingtin inhibits fast axonal transport by activating JNK3 and phosphorylating kinesin. Nat Neurosci (2009) vol. 12 (7) pp. 864-71

[27]Wyttenbach et al. Heat shock protein 27 prevents cellular polyglutamine toxicity and suppresses the increase of reactive oxygen species caused by huntingtin. Human molecular genetics (2002) vol. 11 (9) pp. 1137-51

[28]Kadowaki et al. Amyloid beta induces neuronal cell death through ROS-mediated ASK1 activation. Cell Death and Differentiation (2005) vol. 12 (1) pp. 19-24

[29]Yang et al. Aggregated polyglutamine peptides delivered to nuclei are toxic to mammalian cells. Human molecular genetics (2002) vol. 11 (23) pp. 2905-17

[30]Huang et al. Amyloid formation by mutant huntingtin: threshold, progressivity and recruitment of normal polyglutamine proteins. Somat Cell Mol Genet (1998) vol. 24 (4) pp. 217-33

[31] Kitamura et al. Cytosolic chaperonin prevents polyglutamine toxicity with altering the aggregation state. Nat Cell Biol (2006) vol. 8 (10) pp. 1163-70

[32]Jana et al. Co-chaperone CHIP associates with expanded polyglutamine protein and promotes their degradation by proteasomes. J Biol Chem (2005) vol. 280 (12) pp. 1163540

[33] Bauer et al. Harnessing chaperone-mediated autophagy for the selective degradation of mutant huntingtin protein. Nat Biotechnol (2010) vol. 28 (3) pp. 256-63

[34]Shoji-Kawata et al. Identification of a candidate therapeutic autophagy-inducing peptide. Nature (2013) vol. 494 (7436) pp. 201-6

[35]Jeong et al. Acetylation targets mutant huntingtin to autophagosomes for degradation. Cell (2009) vol. 137 (1) pp. 60-72

[36]Sittler et al. Geldanamycin activates a heat shock response and inhibits huntingtin aggregation in a cell culture model of Huntington's disease. Human molecular genetics (2001) vol. 10 (12) pp. 1307-15

[37]Wang et al. Activation of Hsp70 reduces neurotoxicity by promoting polyglutamine protein degradation. Nat Chem Biol (2013) vol. 9 (2) pp. 112-8

[38]Calamini et al. Small-molecule proteostasis regulators for protein conformational diseases. Nature Chemical Biology (2012) vol. 8 (2) pp. 185-96

[39]Sarkar et al. Small molecules enhance autophagy and reduce toxicity in Huntington's disease models. Nature Chemical Biology (2007) vol. 3 (6) pp. 331-8

[40]Tsvetkov et al. A small-molecule scaffold induces autophagy in primary neurons and protects against toxicity in a Huntington disease model. Proc Natl Acad Sci USA (2010) vol. 107 (39) pp. 16982-7 
[41] Heiser et al. Inhibition of huntingtin fibrillogenesis by specific antibodies and small molecules: implications for Huntington's disease therapy. Proc Natl Acad Sci USA (2000) vol. 97 (12) pp. 6739-44

[42] Heiser et al. Identification of benzothiazoles as potential polyglutamine aggregation inhibitors of Huntington's disease by using an automated filter retardation assay. Proc Natl Acad Sci USA (2002) vol. 99 Suppl 4 pp. 16400-6

[43]Ehrnhoefer et al. Green tea (-)-epigallocatechin-gallate modulates early events in huntingtin misfolding and reduces toxicity in Huntington's disease models. Human molecular genetics (2006) vol. 15 (18) pp. 2743-51

[44]Ehrnhoefer et al. EGCG redirects amyloidogenic polypeptides into unstructured, offpathway oligomers. Nature structural \& molecular biology (2008) vol. 15 (6) pp. 558-66

[45]Scherzinger et al. Huntingtin-encoded polyglutamine expansions form amyloid-like protein aggregates in vitro and in vivo. Cell (1997) vol. 90 (3) pp. 549-58

[46]Andrade et al., HEAT repeats in the Huntington's disease protein. Nature Genetics (1995) vol. 11 (2) pp.115-6

[47]Faber et al., Huntingtin interacts with a family of WW domain proteins. Human Molecular Genetics (1998) vol. 7 (9) pp. 1463-74

[48]Cattaneo et al., Normal huntingtin function: an alternative approach to huntington's disease. Nature Reviews (2005) vol. 6 (12) pp. 919-30

[49]Bilen et al., Drosophila as a Model for Human Neurodegenerative Disease. Annual Reviews (2005) vol. 39 pp. 153-71 
Tabelle 1: Overview of the ten known polyglutamine diseases (modified from [4])

\begin{tabular}{l|c|c|c}
\hline Disease & Protein & $\begin{array}{c}\text { Pathological } \\
\text { repeat length }\end{array}$ & Affected brain region \\
\hline Dentatorubropallidoluysian atrophy (DRPLA) & Atropin-1 & $49-88$ & Cerebral cortex \\
\hline Huntington's disease (HD) & Huntingtin & $40-121$ & Striatum and cortex \\
\hline Spinal and bulbar muscular atrophy (SBMA) & Androgen receptor & $38-62$ & $\begin{array}{l}\text { Motor neurons; brain stem; } \\
\text { spinal cord }\end{array}$ \\
\hline Spinocerebellar ataxia 1 (SCA1) & Ataxin-1 & $39-82$ & Cerebellum \\
\hline Spinocerebellar ataxia 2 (SCA2) & Ataxin-2 & $32-200$ & Cerebellar Purkinje cells \\
\hline Machado-Joseph disease (MJD) & Ataxin-3 & $61-84$ & $\begin{array}{l}\text { Ventral pons and } \\
\text { substantia nigra }\end{array}$ \\
\hline Spinocerebellar ataxia 6 (SCA6) & CACNA1A & $10-33$ & Cerebellar Purkinje cells \\
\hline Spinocerebellar ataxia 7 (SCA7) & Ataxin-7 & $37-306$ & $\begin{array}{l}\text { Cerebellar Purkinje cells; } \\
\text { brain stem; spinal cord }\end{array}$ \\
\hline Spinocerebellar ataxia 12 (SCA12) & PPP2R2B & $66-78$ & $\begin{array}{l}\text { Cerebral and cerebellar } \\
\text { cortex }\end{array}$ \\
\hline Spinocerebellar ataxia 17 (SCA17) & TATA-binding protein & $47-63$ & Cerebellar Purkinje cells
\end{tabular}



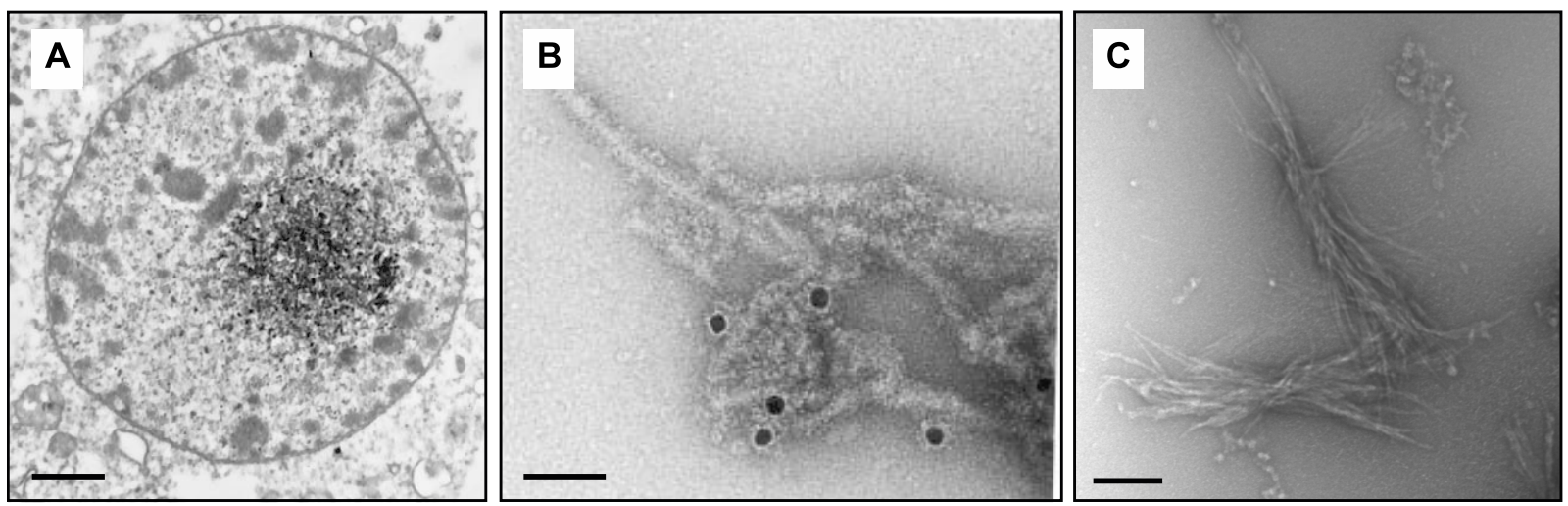

Fig. 1: Electron microscopy of in vivo human nuclear inclusions and in vitro HTTex1 aggregates.

(A) Nuclear inclusion in cortical neurons from a Huntington's disease patient. Scale bar corresponds to $1 \mu \mathrm{m}$. Image by courtesy of DiFiglia et al., 1997 [8].

(B) HTTex1 fibrillar structures isolated from COS-1 cells and immunogold labeled. Scale bar corresponds to $50 \mathrm{~nm}$. Image by courtesy of Scherzinger et al., 1999 [10].

(C) In vitro generated fibrillar HTTex1 aggregates show morphological similarity. Scale bar corresbonds to $100 \mathrm{~nm}$. 
A

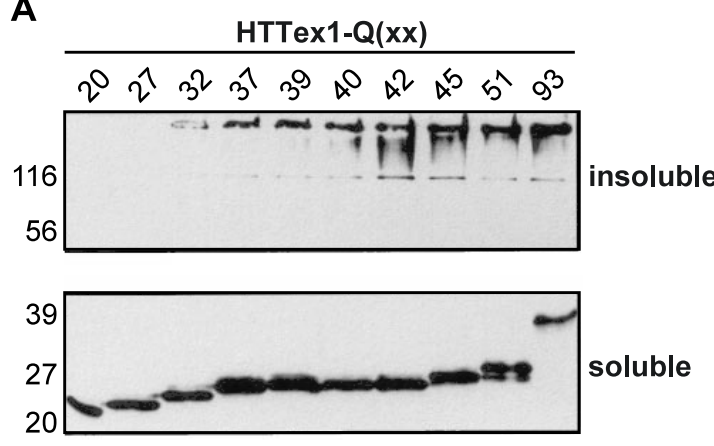

B

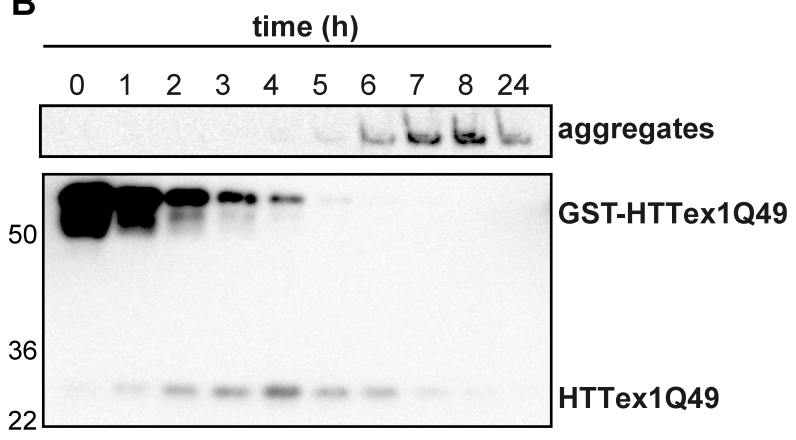

C
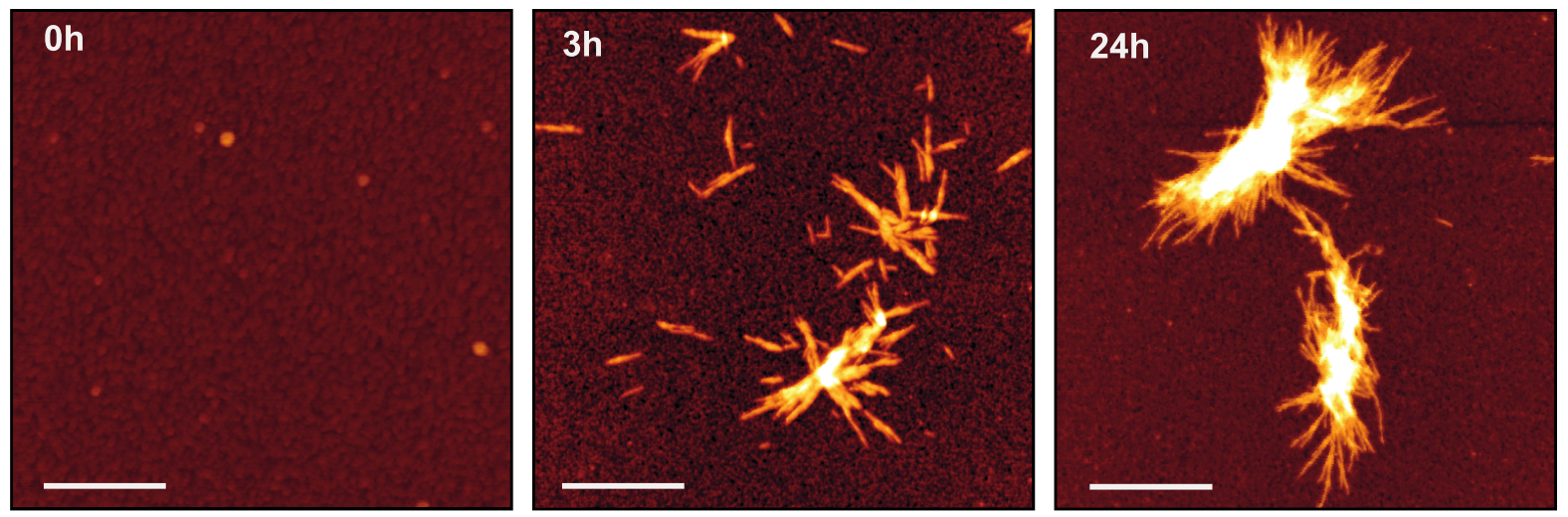

Fig. 2: Spontaneous HTTex1 aggregation is polyQ and time dependent

(A) COS-1 cells expressing HTTex1 fragments with different polyQ lengths form aggregates in a polyQ-length dependent manner. Cell lysates were separated in insoluble (pellet) and soluble (supernatant) fractions by centrifugation. Image by courtesy of Scherzinger et al., 1999 [10].

(B) Proteolytic cleavage of GST-HTTex1Q49 fusion protein results in the release of HTTex1Q49 fragments, which form insoluble aggregates in vitro in a time-dependent manner.

(C) The HTTex1Q49 fragment in a time-dependent manner forms bundles of amyloid-like aggregates with a fibrillar morphology. Aggregation was monitored by atomic force microscopy (AFM). Scale bar corresponds to $500 \mathrm{~nm}$. 
A<smiles>O=C(O[C@H]1Cc2c(O)cc(O)cc2O[C@H]1c1cc(O)c(O)c(O)c1)c1cc(O)c(O)c(O)c1</smiles>

B

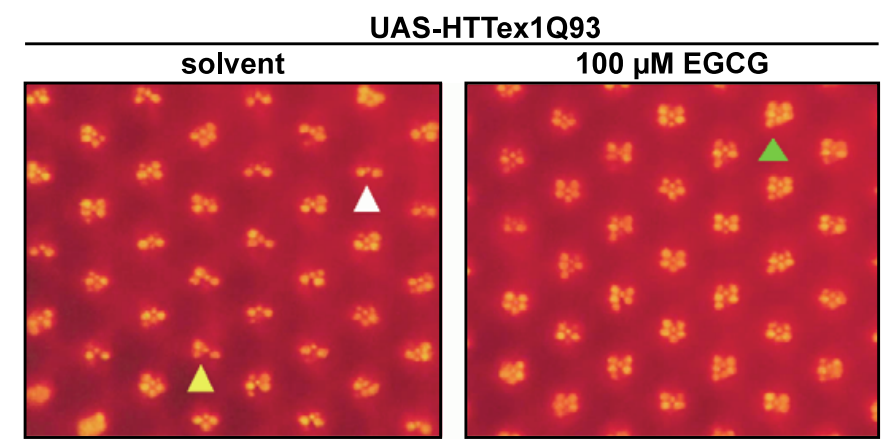

Fig. 3: Figure 3: The compound EGCG protects against HTTex1Q93-induced photoreceptor degeneration in Drosophila melanogaster

(A) Chemical structure of (-)-epigallocatechin-gallate (EGCG).

(B) 7-day old Drosophila melanogaster flies expressing HTTex1Q93 show extensive photoreceptor degeneration. When treated with solvent the ommatidia contain three (white arrowhead) or four (yellow arrowhead) rhabdomers. Flies fed with $100 \mu \mathrm{M}$ EGCG show reduced degeneration, with up to seven rhabdomers (green arrow). Image by courtesy of Ehrnhoefer et al., 2006 [43] 